\title{
Regionale Versorgung krebskranker Kinder und Jugendlicher in der Bundesrepublik Deutschland
}

\author{
G. Landbeck
}

\section{Grundlagen}

\section{Therapieerfolge}

Durch planvoll aufeinanderfolgende Serien prospektiver, kontrollierter und randomisierter Therapiestudienreihen ist bei den im Kindes- und Jugendlichenalter vorkommenden Leukämien und bösartigen Tumoren in den 70er Jahren ein Durchbruch zu hohen Raten langzeitig rückfallfrei Uberlebender gelungen. Die in den pädiatrisch-onkologischen Vereinigungen unseres Landes zusammenarbeitenden Kliniken haben an diesen weltweiten Bemühungen keinen geringen Anteil. Bei optimaler ärztlicher Versorgung kann heute mindestens jedes zweite krebskranke Kind einer Heilung zugeführt werden.

\section{Die Erfolgsverbesserung betrifft}

- akute lymphoblastische Leukämien und hochgradig maligne Non-Hodgkin-Lymphome $(4,7,18)$

- Morbus Hodgkin (20)

- embryonale Tumoren: Neuroblastome (2), WilmsTumoren (3), Rhabdomyosarkome (17)

- Osteosarkome $(21,22,23)$ und Ewing-Sarkome

$$
(8,23,24)
$$

Darüber hinaus beginnen sich deutlich verbesserte Erfolgsaussichten bei akuten myeloischen Leukämien (19), weiteren Weichteilsarkomen und auch beim Medulloblastom abzuzeichnen.

\section{Bausteine des Therapieerfolges}

Jeder entscheidende Fortschritt ist bislang durch rationale und konsequente Nutzung klinischer Beobachtungen und Erkenntnisse erzielt worden, im wesentlichen also empirischer Natur. Das betrifft die Entwicklung spezieller Anwendungsweisen bekannter und neuer Zytostatika wie auch die sinnvolle Einfügung operativer und strahlentherapeutischer Maßnahmen.

Die Kombination einer intensivierten, systemisch wirkenden Polychemotherapie mit einer stetig verbesserten lokalen operativen und/oder strahlentherapeutischen Behandlung liegt jedem erfolgreichen Therapiekonzept zugrunde. Erfolge setzen jedoch spezielle Erfahrungen und ein abgestimmtes Zusammenwirken, ein Miteinander aller beteiligten Fachdisziplinen auf diagnostischem und therapeutischen Gebiet voraus.
Die Früherkennung (fälschlicherweise oft als Vorsorge be nannt) bzw. die Zunahme früher Verdachtsdiagnosen durch niedergelassene Ärzte und erstversorgende Krankenhäuser ist proportional zum Behandlungserfolg angestiegen und damit gleichzeitig die Primärzuweisung an ein kompetentes Behandlungszentrum (14). Diese Erfahrungen sind von grundsätzlicher Bedeutung, weisen sie doch auf den fragwürdigen Sinn einer frühen Diagnose bei fehlenden Erfolgsaussichten, wie auch auf die Notwendigkeit einer vertrauenswürdigen, optimal ausgeruisteten Spezialeinrichtung im regionalen Bereich. Krebsfrüherkennung ist nur begrenzt eine Frage der öffentlichen Aufklärung. Sie ist vielmehr noch eine Funktion des Abbaues ärztlicher Hilflosigkeit, und zwar sowohl auf therapeutischem Gebiet als auch in der Organisation einer umfassenden Versorgung (siehe hierzu auch 14).

\section{Nebenwirkungen der Behandlung}

Vor allem die Strahlentherapie und Chemotherapie sind mit unerwünschten Folgen belastet.

Keines der vielen heute verwendeten Zytostatika hat eine echte Tumorspezifität. Sie alle treffen Krebszellen wie auch Normalzellen, wobei erstere in stärkerem Maße geschädigt werden. Diese Differenz bestimmt den Therapieerfolg. Die Intensivierung der Polychemotherapie mußte zwangsläufig zu erheblichen Nebenwirkungen mit potentiell lebensbedrohlichen Folgen führen. Sie ist so auch erst durch die Entwicklung und Verfugbarkeit gezielter therapeutischer Gegenmaßnahmen zusammen mit einschlägigen ärztlichen und pflegerischen Erfahrungen verantwortbar geworden. Als Maß der individuellen therapeutischen Belastung gilt die gerade noch mögliche Steuerbarkeit unerwünschter Wirkungen, solange keine hohen Raten langzeitig rückfallfrei Uberlebender erreicht worden sind bzw. bei Therapiebeginn im Einzelfall noch kein unterschiedliches Krankheitsrisiko mit Sicherheit erkennbar ist.

\section{Seltenes Vorkommen von Krebskrankheiten bei Kindern und Jugendlichen}

Die Morbiditätsrate in dieser Altersgruppe ist gegenüber Erwachsenen gering. Sie liegt bei 12/100.000 Kinder und Jahr bzw. bei 29/1 Million Bevölkerung und Jahr. In der Bundesrepublik Deutschland sind entsprechend jährlich etwa 1.800 Neuerkrankungen zu erwarten $(13,14)$. Der

Frau Prof. Dr. Hedwig Wallis zum 60. Geburtstag gewidmet. 
heute hohe Anteil langzeitig Uberlebender hat jedoch eine Kumulation langfristig intensiv $\mathrm{zu}$ versorgender und $\mathrm{zu}$ überwachender krebskranker Kinder und Jugendlicher bewirkt. Sie beläuft sich auf eine Größe von 9.000-10.000 Patienten pro Jahr.

\section{Folgerungen}

Nach dem gegenwärtigen Stand der Erfolgsentwicklung in der Behandlung krebskranker Kinder und Jugendlicher ist jede Therapieplanung auf ein kuratives Ziel zu richten.

Heilungen sind nach wie vor jedoch fast nur auf dem Wege schwerster therapeutischer Belastungen, vor allem mit unspezifisch wirkenden und nebenwirkungsreichen Pharmaka zu erzielen. Die Optimierung ihrer Anwendung und Verbesserungen in der Begegnung ihrer unerwünschten Wirkungen werden somit auch den weiteren Fortschritt bestimmen. Solange aber Krebskrankheiten nicht zuverlässig und unter akzeptablen Behandlungsrisiken geheilt werden können, sind diese Behandlungsverfahren jeweils als vorläufig und ihrem Wesen nach als experimentell einzustufen.

Unterstellen wir, daß jedem krebskranken Kind auch unter diesen Voraussetzungen grundsätzlich das Recht auf einen jeweils bestmöglichen Behandlungserfolg einzuräumen ist, jeder Patient also an der weiteren Erfolgsentwicklung teilhaben soll, so ist diese Forderung nur erfullbar und ärztlich vertretbar, wenn die Behandlung ausschließlich im Rahmen umfassend protokollierter prospektiver Therapiestudienreihen vorgenommen wird. Krebstherapie ist unabwendbar zur forschenden Behandlung geworden.

Das seltene Vorkommen der Krebskrankheiten im Kindesund Jugendlichenalter wie auch das notwendige ungewöhnlich hohe $\mathrm{MaB}$ an ärztlicher und pflegerischer Erfahrung in der Steuerung der Therapie und ihrer Nebenwirkungen zwingen zu einer regional weitrüumigen Zentralisierung der Patientenversorgung in speziellen Behandlungseinrichtungen, ist doch die Frage der Kompetenz in jedem Einzelfall zu einem lebensentscheidenden Faktor geworden. In diesem Sinne ist als Mindestmaß einer regionalen Zentralisierung eine Zahl jährlicher Neuzugänge von 70 , besser jedoch von 100-120 Patienten geboten. Das entspricht einer Bevölkerungsgruppe von ca. 3 Millionen bzw. 4-5 Millionen Einwohnern. Daruber hinaus ist es selbst bei Zentralisierungen dieser Größenordnung unumgänglich, prospektive Therapieprojekte mit kooperativer Beteiligung vieler regio. naler Zentren zu planen und durchzuführen, um jeweils in kurzer Zeit zureichende Patientenzahlen und sichere Aussagen über den Studienverlauf zu erhalten. (Ausführliche Darstellungen zur Organisation einer optimalen Versorgung krebskranker Kinder in der Bundesrepublik Deutschland siehe $13,14,15,16)$.

Können wir also davon ausgehen, daß die Erfolge der Krebstherapie im Kindes- und Jugendlichenalter nur dann unter optimalen Bedingungen erzielt und weiterentwickelt werden können, wenn jede Behandlung im Rahmen multizentrischer klinisch-wissenschaftlicher Projekte durchgeführt wird, so ist aber auch festzustellen und gegenüber der Onkologie des Erwachsenen deutlich hervorzuheben, daß prospektive Therapiestudienreihen im Kindes- und Jugendlichenalter zur Regelversorgung geworden sind, und daran wird sich in absehbarer Zeit nichts ändern. Dieser Sonderheit der klinischen Onkologie im Kindes- und Jugendlichenalter gebührt hohe Aufmerksamkeit, ergeben sich doch unausweichliche und weitreichende Folgen für alle Leistungsträger, die zur Sicherstellung einer optimalen Krankenversorgung im stationären und ambulanten Bereich verpflichtet sind.

\section{Stand der Entwicklung in der Bundesrepublik Deutschland}

\section{Wissenschaftliche Vereinigungen}

Die Entwicklung und Organisation der klinischen Onkologie des Kindesalters in unserem Lande ist im wesentlichen ein Verdienst hämatologisch-onkologisch tätiger Pädiater und im engsten Sinne mit der ersten Vereinsgründung dieses Ärztekreises, der Deutschen Arbeitsgemeinschaft fir Leukämie-Forschung und-Behandlung im Kindesalter e.V. (DAL) im Jahre 1965 verbunden. Sie hat heute über 240 Mitglieder; alle Universitätskinderkliniken sind vertreten. Der Vorstand der Arbeitsgemeinschaft gründete 1973 nach Verhandlungen mit den Vorständen aller an der Versorgung krebskranker Kinder beteiligten Fachgesellschaften die Gesellschaft für Pädiatrische Onkologie e.V. (GPO) als interdisziplinären Dachverband, um so die nötigen Voraussetzungen für ihre aktive, paritätische Wirkung in der Weiterentwicklung der klinischen Onkologie des Kindesund Jugendlichenalters zu schaffen. Beide Vereinigungen veranstalten halbjährlich öffentliche Arbeitssitzungen (12)

Alle seit Anfang der 70er Jahre begonnenen, multizentrischen prospektiven Therapiestudienreihen (Phase II- und Phase III-Studien) in der Bundesrepublik sind von diesen Vereinen auf den Weg gebracht worden, wobei eine Arbeitsteilung derart vorgenommen wurde, daß primär disseminierte Krebskrankheiten in den Arbeitsbereich der DAL und solide Tumoren in den der GPO fallen. Die Studienplanung und Protokollabfassung obliegt jeweils kompetenten Vertretern aller involvierten Fächer, die äußerst aufwendige, schwierige und verantwortungsreiche zentrale Projektleitung in der Regel einem pädiatrischen Onkologen. Solche Leitungsfunktionen können erfahrungsgemä $B$ nur für ein bis höchstens zwei multizentrische Therapiestudien von einer klinischen Einrichtung getragen werden, so daß sich notwendigerweise eine breite Verteilung dieser Aufgaben ergibt. Bei der Vielzahl multizentrischer Projekte ist damit aber auch vielen Kollegen die Möglichkeit gegeben, intensive organisatorische Erfahrungen in der klinischen Forschung zu sammeln, was zuvor kaum gegeben war und der Arbeitsweise wissenschaftlicher klinischer Einrichtungen grundsätzlich zugute kommen dürfte. Die an einem Projekt mitarbeitenden $\AA$ rzte treffen sich mindestens einmal jährlich, Zwischenergebnisse des Verlaufs einer Therapiestudie werden in der Regel einmal im Jahr anläßlich der Vereinstagungen öffentlich vorgelegt und publiziert. 


\section{Zentrale Referenzeinrichtungen und Register}

Prospektive Therapiestudien erfordern - nicht zuletzt auch bei der Seltenheit der einzelnen Krebskrankheiten im Kindes- und Jugendlichenalter - eine sichere Diagnose, einheitliche Klassifizierung und risikoorientierte Subtypisierung der Leukämien und Tumoren. Während die Zentralisierung zytomorphologischer, zytochemischer und in den letzten Jahren auch immunzytologischer Differenzierungen der Leukämien von einzelnen pädiatrisch-onkologischen Abteilungen bereits seit vielen Jahren wahrgenommen wird, war es ein unerläßliches Erfordernis, ein Referenzzentrum für klinisch-pathologische Verbundstudien auf dem Gebiet bösartiger Tumoren einzurichten. Mit Hilfe der Stiftung Volkswagenwerk gelang es 1976, dieses bereits 1973 geplante Vorhaben als Zentrales Kinder-Tumorregister an der Abteilung für Paidopathologie des Instituts für Pathologie der Universität Kiel unter der Leitung von Prof. Dr. D. Harms zu verwirklichen $(5,6)$. Die wissenschaftliche Auswertung des Gewebsmaterials erfolgt in enger und direkter Zusammenarbeit mit den regional zuständigen Pathologen.

Mit dem Aufbau einer Dokumentationszentrale zur Erfassung aller Krankheitsfälle in der Bundesrepublik (Morbiditätsregister), für statistische Beratungen in der Projektplanung und Protokollabfassung wie auch einer studienbegleitenden Registrierung aller wichtigen patientenbezogenen Ersterhebungs- und Verlaufsdaten, wurde 1979 nach gehöriger Vorarbeit und mit Hilfe erheblicher Drittmittel am Institut für Medizinische Dokumentation und Statistik der Universität Mainz unter der Leitung von Prof. Dr. J. Michaelis begonnen (Kooperative Dokumentation Malignome im Kindesalter) (9).

\section{Aufbau regionaler Versorgungszentren}

Der frühe Zusammenschluß und die erfolgreich geübte kontinuierliche Zusammenarbeit pädiatrischer Onkologen, die folgerichtige Einbeziehung aller beteiligten Fachdisziplinen in die Verbandsarbeit, die Gründung zentraler Register, die erfolgreiche Durchführung multizentrischer prospektiver Therapiestudienreihen, wie auch eine Vielzahl publizierter Beiträge zur Organisation einer optimalen, umfassenden Versorgung krebskranker Kinder und Jugendlicher in der Bundesrepublik Deutschland bezeugen jahrelange, planvolle und nicht erlahmende ärztliche Bemühungen um eine stetige Verbesserung der Krebsbekämpfung in unserem Lande $(10,11,12,13,14,15,16)$.

Sind uns auch wichtige Hilfen in der Kontaktaufnahme mit renommierten ausländischen Behandlungszentren (KindPhilipp-Stiftung), in der apparativen Grundausstattung (Leukämie-Forschungs-Hilfe e.V.) und zur Verbesserung der regionalen Versorgung krebskranker Kinder (einmalige Mittelzuwendung an 5 pädiatrisch-onkologische Abteilungen durch die Deutsche Krebshilfe e.V., Aktion der Zeitschrift HORZU) zuteil geworden, so müssen wir doch feststellen, daß es bislang nicht gelungen ist, unser, in den Vereinssatzungen festgelegtes Ziel einer optimalen Versorgung jedes krebskranken Kindes, also eine flächendeckende Versorgung zu erreichen. Das ist in Anbetracht der der- zeitig möglichen Behandlungserfolge eine bedrückende Erkenntnis, geht es doch in jedem Einzelfall um Tod oder Uberleben.

Um das Ziel einer flächendeckenden Versorgung aller krebskranken Kinder und Jugendlichen zu erreichen, bedarf es bei einer Zahl von 1.800 Neuerkrankungen pro Jahr der Einrichtung von ca. 15-20 regionalen Behandlungszentren. Den Forderungen nach einer Mindestzahl von jährlich 70-100 Neuzugängen pro Zentrum wie auch der Vermeidung z.T. besorgniserregender langer Anfahrtswege in der langzeitigen ambulanten Versorgung wäre damit genüge getan. In der Bundesrepublik gibt es bislang jedoch nur an 7 Universitäts-Kinderkliniken hämatologischonkologische Spezialabteilungen, die zumindest die strukturellen Grundbedingungen eines regionalen Behandlungszentrums erfüllen. Zu den 5 Anfang der 70er Jahre gegründeten Abteilungen sind in den letzten Jahren somit nur 2 hinzugekommen. Diese 7 Abteilungen versorgen zusammen ca. 25-30\% der krebskranken Kinder und Jugendlichen unseres Landes. Weitere Universitätskliniken und große Kinderkrankenhäuser beteiligen sich an einer protokollgerechten Versorgung Krebskranker und verfügen über speziell ausgebildete und erfahrene Ärzte. Die hier aufgrund erheblicher Intensivierung der nebenwirkungsreichen Therapie, aufwendiger Diagnostik und sehr viel schwieriger gewordenen Führung der Patienten heute notwendigen strukturellen Voraussetzungen im räumlichen und personellen Bereich konnten jedoch bislang noch nicht erfült werden, was die Bewältigung der täglichen hohen Leistungsanforderungen auf ärztlichem, pflegerischen und medizinisch-technischen Gebiet außerordentlich erschwert und auch den Behandlungserfolge infrage stellen kann.

Dieser bedenkliche, nicht unerhebliche Rückstand in der Entwicklung einer bundesweiten optimalen Versorgung krebskranker Kinder und Jugendlicher kann sicherlich kaum auf mangelhafte ärztliche Einsicht zurückgeführt werden. Auch ist in restriktiven Haushaltsplanungen nur dann eine Begründung zu sehen, wenn unterstellt werden kann, daß Ausmaß und Art des Mißstandes den verantwortlichen Entscheidungsgremien bekannt sind. Wichtig erscheint es jedoch, für künftige Planungen zur Kenntnis zu nehmen, daß die stürmische Erfolgsentwicklung der pädiatrischen Onkologie in den letzten Jahren nur dann für alle Betroffenen zum Tragen kommen kann, wenn man erkennt

- daß mögliche Behandlungserfolge nur durch äußerst aufwendige, vor allem personalintensive Therapieverfahren und unter optimalen räumlichen Bedingungen sichergestellt werden können,

- daß weitere Erfolgsverbesserungen nur auf dem Wege kooperativer, multizentrischer prospektiver Therapieprojekte zu erreichen sind,

und schließlich

- daß auf absehbare Zeit eine optimale Regelversorgung jedes Einzelfalles nur durch Einbeziehung in laufende prospektive Therapieprojekte gegeben ist.

Schließlich bleibt anzumerken, daß eine sorgfältige Planung gut über das Land verteilter und ausgerüsteter regionaler Behandlungszentren gegenüber der derzeitig noch über- 
wiegend weitläufig disseminierten Versorgung auch eine finanziell wesentlich ökonomischere Lösung sein dürfte.

\section{Aktuelle Probleme der regionalen Versorgung}

\section{Therepieplanung und klinikbegleitende Forschung}

Die Rekrutierungsphase einer prospektiven Therapiestudie sollte grundsätzlich nicht länger als 2 Jahre in Anspruch nehmen, um nicht in die Gefahr zu geraten, Patienten womöglich einem überholten Behandlungskonzept zuzuführen. In diesem Rhythmus erfolgen Neuplanungen, von denen weitere Fortschritte im Therapieerfolg erwartet werden. Nach dem derzeitigen Stand erreichbarer Heilungsraten können Fortschritte jedoch nicht mehr allein an wachsenden Zahlen rückfallfrei tberlebender gemessen werden. Nicht minder wichtig ist es geworden, die Behandlungsintensität am individuellen Krankheitsrisiko zu orientieren, d.h. soweit bei Diagnosestellung erkennbar, ein hohes und niedriges Risiko vom Standardrisiko zu unterscheiden, um das Maß der therapeutischen Belastung, der Therapiemorbidität und möglichen Spätfolgen der Behandlung ohne Erfolgseinbuße in jeweils vertretbaren Grenzen zu halten. Initial erkennbare Risikofaktoren ergeben sich z.T. aus retrospektiven Analysen klinischer Daten therapeutischer Studienreihen. Nicht minder wichtig haben sich in dieser Hinsicht wie auch zur Erkennung der Qualität einer kompletten Remission zytomorphologische, zytochemische, immunzytologische, biochemische und molekularbiologische Studien erwiesen, die in der Regel nur dort erwartet werden können, wo entsprechende Materialmengen anfallen, d.h. in regionalen Behandlungszentren. So ist Versorgung über prospektive Therapiestudien, also über klinische Forschung hinaus, auch grundsätzlich mit klinikbegleitenden Forschungscufgaben verbunden, und man kommt kaum umhin, hierin einen sozialen Auftrag zu sehen. Regionale Versorgungszentren sollten daher gleichzeitig auch wissenschaftliche Einrichtungen sein und räumlich so ausgestattet werden, daß sie dieser Auflage nachkommen können.

\section{Verbesserung der stationären Behandlung}

Soweit im pädiatrisch-onkologischen Bereich stationäre Aufenthalte erforderlich sind, handelt es sich in der Regel um Patienten mit aufwendiger Primärdiagnostik, um Beginn der konservativen Krebstherapie in der postoperativen Phase, gefahrvolle Infusionen hochdosierter Zytostatika, Behandlung lebensbedrohlicher Nebenwirkungen der Krebstherapie, Behandlung schwerer und komplizierender Zweiterkrankungen, risikoreiche Behandlungen von Krankheitsrückfallen und nicht zuletzt auch finale Krankheitsstadien. Eine optimale ärztliche und pflegerische Versorgung dieser Patienten erfordert ein hohes Maß spezieller Kenntnisse und Erfahrungen, das nur dann gegeben ist und wachsen kann, wenn man sich täglich und überwiegend mit diesen Problemen auseinandersetzen muB.
So ist es auch unausweichlich, die stationäre Versorgung krebskranker Kinder auf einer eigens hierfür vorgesehenen und eingerichteten Bettenstation vorzunehmen und dafur Sorge zu tragen, daß ein Personalwechsel, soweit erforderlich, nur in größeren Zeitabständen vorgenommen wird. Die Kontinuität der pflegerischen Versorgung sollte durch Funktionsschwestern getragen werden.

Die Mitaufnahme der Mütter ist nicht nur aus psychischen Gründen geboten. Sie hat sich für die notwendige kundige Mitarbeit besonders auch in der ambulanten Versorgung, für das Verständnis dieser komplizierten und nebenwirkungsreichen Therapieverfahren als unverzichtbare Regelung erwiesen, ganz abgesehen von der pflegerischen Entlastung, die unsere hart arbeitenden und schwergeprifften Schwestern erfahren.

Wer auch heute noch krebskranke Patienten über alle Stationen eines Hauses verteilt in der Vorstellung, daß es menschlich unzumutbar ist, diese Schwerbetroffenen zusammenzulegen, sollte bedenken, daß ein „Ghetto" seit jeher auch ein hohes Maß an organisiertem Schutz bedeutet.

\section{Verbesserung der ambulanten Versorgung}

Die Chemotherapie führt unvermeidbar zu einer stark verminderten Infektionsabwehr des Patienten und zwingt zur Meidung stationärer Aufenthalte, um schwerwiegenden Hospitalinfektionen mit Problemkeimen zu entgehen. Die vorwiegend ambulante Versorgung ist im wesentlichen hierin begründet, wenngleich auch der chronische Krankheitsverlauf für sich genommen genuigend $A$ la $B$ ist, die Patienten möglichst kontinuierlich in der Familie, in ihrer gewohnten Umgebung zu belassen. Das hat zur Folge, daß eingreifende diagnostische und therapeutische Maßnahmen ambulant vorgenommen werden und Patienten und Angehörige erheblich belasten. So ist es nötig, Eltern in die Symptomatologie der Krankheit, Besonderheiten des Krankheitsverlaufes, in Therapieerwartungen und Nebenwirkungen sowie pflegerische Aufgaben rückhaltlos einzuweisen, um eine aktive Mitarbeit zu ermöglichen. Die Ambulanz eines regionalen Behandlungszentrums muB daher über eine ausreichende Zahl an Räumen, vor allem auch an Isolierräumen für infektiöse Kinder (i.B. Varizellen, Zoster), und erfahrenes Personal verfuigen, um diesen besonderen und zeitaufwendigen Auflagen nachkommen zu können.

Erfordert eine protokollgerechte Durchführung prospektiver Therapiestudienreihen mit nicht sicher voraussehbaren und wägbaren Risiken notwendigerweise eine ambulante Versorgung durch das Behandlungszentrum, so ist diese Ausschließlichkeit nach AbschluB intensiver Behandlungsphasen nicht mehr in engem Maßse gegeben, soferm Hausärzte und wohnortnahe Krankenhäuser bereit und imstande sind, an einer protokollgerechten Versorgung partnerschaftlich und verantwortlich mitzuwirken. Bedenkt man, welche Entfernungen vom Wohnort zum Behandlungszentrum jeweils zurückzulegen sind (der Durchschnitt bei den vom Hamburger Behandlungszentrum betreuten Patienten beträgt $75 \mathrm{~km}$ !), so wird deutlich, daß eine partiell dezentralisierte Versorgung prinzipiell unver- 
zichtbar ist. Sie kann jedoch nur dann verwirklicht werden, wenn Sicherheit und Qualität der durchaus noch risikorejchen Dauerbehandlung nicht gefährdet sind. Zwei Voraussetzungen müssen dazu erfüllt sein, nämlich regelmäßige gezielte Fortbildungen im regionalen Einzugsgebiet eines Behandlungszentrums sowie eine zügige und vollständige Datenvermittlung. Diese wird auf konventionellem Wege (Arztbrief) nicht erreicht und kann nur über ein Rechnergestütztes Informationssystem, das alle Daten der breitangelegten Primär- und Verlaufsdiagnostik wie auch der Therapie speichert und jederzeit verfugbar macht, gewährleistet werden. Die Einrichtung und Förderungswürdigkeit solcher Projekte verdient Aufmerksamkeit. Die Entwicklungsphase der ersten klinischen Informationssysteme wird in Kürze abgeschlossen sein.

\section{Verbesserungen des Stellenplanes regionaler Behandlungszentren}

Durch Anwendung der Anhaltszahlen der Deutschen Krankenhausgesellschaft von 1969 werden die zur stationären wie ambulanten Versorgung krebskranker Kinder und Ju. gendlicher benötigten Planstellen auch nicht annähernd erreicht. Das betrifft alle Personalbereiche, besonders jedoch das Pflegepersonal, zumal auf diesem Sektor in der Regel noch nicht einmal vorübergehend Drittmittel verfügbar sind. Der Personalstellenmangel gefährdet Patienten und weist nachdrücklich auf die bisher unzureichende Versorgungsplanung im regionalen Bereich. Die Uberlastung der Schwestern und Ärzte mit schwierigen und verantwortungsreichen Aufgaben führt darüber hinaus zu erheblichen Personalfluktuationen, die eine kontinuierliche kompetente Versorgung kaum entstehen läßt.

Die Gesellschaft für Pädiatrische Onkologie hat daher 1978, nach gründlicher Prüfung des täglichen Arbeitsaufwandes in den bislang etablierten regionalen Versorgungszentren, ein Memorandum zur personellen Situation in der pädiatrischen Onkologie verabschiedet und eine Berechnung des Personalbedarfs im diagnostischen und therapeutischen Bereich vorgelegt (11). Die erarbeiteten Zahlen entsprechen im stationären Pflegedienst den Ansätzen für Intensivüberwachungseinheiten. Anhaltszahlen für Ärzte und technisches Personal wie auch Personalzahlen für den ambulanten Sektor sind aufgrund sorgfältiger Analysen erstellt worden. Diese Personalbedarfszahlen wurden 1979 im vollen Umfang auch von der Arbeitsgemeinschaft Deutscher Tumor. zentren übernommen (1). Es dürfte kein Zweifel darin bestehen, daß die weitere Entwicklung der regionalen Versorgung im engen Maße von der Erfüllung berechtigter Forderungen in der personellen Grundausstattung abhängt. Sie ist schließlich auch eine wesentliche Voraussetzung für die Bereitstellung finanzieller Förderungen bei multizentrischen prospektiven Therapieprojekten.

\section{Schlußbemerkungen}

Die rasche Erfolgsentwicklung der pädiatrischen Onkologie hat $\mathrm{zu}$ einer Fülle ernsthafter und noch unbewältigter Pro- bleme in der regionalen Versorgung krebskranker Kinder und Jugendlicher geführt, um jedem Betroffenen die gleiche Chance zu geben. Das betrifft den flächendeckenden Aufbau und Ausbau regionaler Spezialeinrichtungen mit begründeten und nicht unerheblichen Forderungen in der räumlichen, personellen und apparativen Ausstattung, wobei diese organisatorischen Regelungen letztlich nicht nur die Basis jeder weiteren Erfolgsentwicklung sind, sondern auch die ökonomischste Lösung der Problembewältigung wären.

Da jede Behandlung aufgrund der besonderen Gegebenheiten der pädiatrischen Onkologie praktisch nur im Rahmen multizentrischer Therapieprojekte zu einem optimalen Erfolg führen kann, ist die Beteiligung möglichst vieler regionaler Versorgungseinrichtungen unverzichtbar. Die Mitarbeit an prospektiven Therapiestudien ist von der zentralen Studienleitung jedoch nur dann verantwortbar, wenn diese protokollgerecht und unter Wahrung einer kontinuierlichen tbermittlung der benötigten Verlaufsdaten erfolgt. Andernfalls muß mit unvertretbaren Gefährdungen der Patienten gerechnet werden. Auch diese Auflage einer Regelversorgung auf onkologischem Gebiet setzt funktionstüchtige regionale Einrichtungen voraus.

Bei der relativ kleinen Zahl krebskranker Kinder und Jugendlicher ist es selbst in Anbetracht der zu erbringenden speziellen und äußerst aufwendigen Leistungen nicht vertretbar, analog der Erwachsenenonkologie einen Facharzt für pädiatrische Onkologie bzw. pädiatrische Hämatologie und Onkologie zu fordern. Der Schwerpunkt der Versorgung wird immer in regionalen klinischen Zentren liegen, so daß für den niedergelassenen Arzt eine, wenn auch dringend notwendige Mitarbeit verbleibt. Entsprechend muß die onkologische Ausbildung und Fortbildung einerseits auf alle Kinderärzte und Kollegen involvierter weiterer Fachdisziplinen gerichtet sein und zum anderen dafuir Sorge tragen, daß Ausbildungsplätze in den Spezialabteilungen eingerichtet werden, um Spezialisten für den gewachsenen Bedarf an regionalen Behandlungszentren wie auch Nachwuchskräfte zur Deckung des fortlaufenden Personalbedarfs dieser Einrichtungen heranzubilden. Das betrifft Ärzte, Schwestern und medizinisch-technische Assistenten.

\section{Literatur}

1 Arbeitsgemeinschaft Deutscher Tumorzentren: Zur Konzeption und zum Personal- und Finanzierungsbedarf der Tumorzentren in der Bundesrepublik Deutschland, 2. Aufl. Heidelberg 1979

2 Berthold, F., J. Treuner, D. Niethammer, F. Lampert: Neuroblastomstudie $\mathrm{NBL}_{70}$ der Gesellschaft für Pädiatrische Onkologie. Zwischenbericht nach 1 Jahr. Erg. Päd. Onk. 5 (1981)

3 Gutjahr, P.: Wilms-Tumoren - Eine Standortbestimmung. Erg. Päd. Onk. 5 (1981)

4 Haas, R.J., G. Janka, B. Netzel, M. Helmig: Münchener Studie zur Behandlung der akuten lymphoblastischen Leukämie im Kindesalter (ALL 77-01). Erg. Päd. Onk. 5 (1981)

5 Harms, D., K. Lennert: Zur Frage des zentralen Tumorregisters der Gesellschaft. Erg. Päd. Onk. 2 (1978) 5

6 Harms, D., J. Gottschalk, H. Griessner, J. Hedderich, H. Wilke, $E$. Wilke: Zentrales Tumorregister bei der Gesellschaft für Pädiatrische Onkologie e.V. - erster Erfahrungsbericht. Erg. Päd. Onk. 4 (1980) 5 
7 Henze, G., H.J. Langermann, H. Gadner, G. Schellong, K. Welte, H. Riehm: Ergebnisse der Studie BFM 76/79 zur Behandlung der akuten lymphoblastischen Leukämie bei Kindern und Jugendlichen. Erg. Päd. Onk. 5 (1981)

8 Jürgens, H.: Ewing-Sarkom bei Kindern und Jugendlichen: Planung einer kooperativen Therapiestudie der Gesellschaft für Pädiatrische Onkologie (CESS 81). Erg. Päd. Onk. 5 (1981)

9 Kaatsch, P., J. Michaelis: Das kooperative Dokumentationsprojekt für kindliche Malignome. Erg. Päd. Onk. 5 (1981)

10 Kornhuber, B.: Zur Situation der pädiatrischen Onkologie in der Bundesrepublik. Erg. Päd. Onk. 2 (1978) 1

11 Kornhuber, B.: Stellungnahme der Geselischaft für Pädiatrische Onkologie e.V. zur personellen Situation in der pädiatrischen Onkologie der Bundesrepublik Deutschland. Erg. Päd. Onk. 3 (1979) 133

12 Landbeck, G.: 10 Jahre Deutsche Arbeitsgemeinschaft furr Leukämie-Forschung und -Behandlung im Kindesalter e.V. Erg. Päd. Onk. 1 (1977) 1

13 Landbeck, G.: Einfühungsreferat: Tumoren im Kindesalter. In: A. Georgii, 13. Deutscher Krebs-Kongreß 1976. G. Fischer 1978

14 Landbeck, G.: Zur Organisation einer optimalen Versorgung krebskranker Kinder in der Bundesrepublik Deutschland. Erg. Päd. Onk. 3 (1979) 1

15 Landbeck, G.: Entwicklung einer regional zentralisierten Versorgung krebskranker Kinder im Raum Hamburg. Kinderarzt 12 (1981) 105

16 Landbeck, G.: Pädiatrische Onkologie. Kinderarzt 12 (1981) 161
17 Maurer, H.M., T. Moon, M. Donaldson und Mitarb.: The Intergroup Rhabdomyosarcoma Study - Update November 1978. Nat. Cancer Inst. Monogr. 1979

18 Riehm, H., H. Gedner, K. Welte: Die West-Berliner-Studie zur Behandlung der akuten lymphoblastischen Leukämie des Kindes - Erfahrungsbericht nach 6 Jahren. Erg. Päd. Onk. 1 (1977) 89

19 Scheer, K., G. Schellong, H. Riehm: Verbesserte Prognose der akuten myeloischen Leukämien bei Kindern nach intenstver Anfangstherapie. Erg. Päd. Onk. 3 (1979) 104

20 Schellong, G.: Bericht tiber die Hodgkin-Therapie-Studie HD 78. 30. Tagung der Arbeitsgemeinschaft für LeukämieForschung und -Behandlung im Kindesalter e.V. Frankfurt 13.6.1980

21 Winkler, K., W. Becker, R. Kotz: Presentation of the collaborative osteosarcoma study COSS 77. Chimioth. oncol. 2 (1978) 225

22 Winkler, K., I. Grosch-Wörner, I. Luy-Harm, G. Marsmann, J. Miller, G. Landbeck: Zur adjuvanten Chemotherapie des Osteosarkoms. 3 Jahre Erfahrung mit hochdosiertem Methotrexat/Citrovorum-Factor-Rescue, Adriblastin und Endoxan. Z. Kinderchir. 26 (1979) 293

23 Winkler, K., G. Landbeck: Die Behandlung maligner Knochentumoren. Pharmakotherapie 3 (1980) 199

25 Winkler, K., G. Landbeck: Zur Chemotherapie des EwingSarkoms. Erg. Päd. Onk. 5 (1981)

25 Winkler, K., E. Matzke, S. Caspers, R. Erttmann, I. GroschWörner, H. Kabisch, J. Müller, G. Landbeck: Vorstellung der kooperativen Studie COALL-80 zur Behandlung der akuten lymphoblastischen Leukämie (ALL) im Kindesalter. Erg. Päd. Onk. 5 (1981)

Prof. Dr. G. Landbeck, Tumorzentrum Hamburg, Abteilung für Hämatologie und Onkologie der Universitäts-Kinderklinik Hamburg, Martinistraße 52, 2000 Hamburg 20 